Anestesiología

Abril-Junio 2021

Vol. 44. No. 2. pp 91-97

doi: $10.35366 / 99011$

\section{Protocolo para el manejo anestésico del binomio materno fetal en el proyecto EXIT para neonatos con hernia diafragmática}

\author{
Protocol for the anesthetic management of the maternal-fetal binomial \\ in the EXIT project for neonates with diaphragmatic hernia
}

\author{
Dr. Víctor Edmundo Fuentes-García,* Dr. Juan Manuel Alarcón-Almanza, ${ }^{\ddagger}$ \\ Dra. Kelly Arlett Maldonado-Sánchez, ${ }^{\ddagger}$ Dra. Nathalie Contreras-Ramírez ${ }^{\S}$
}

\begin{abstract}
Citar como: Fuentes-García VE, Alarcón-Almanza JM, Maldonado-Sánchez KA, Contreras-Ramírez N. Protocolo para el manejo anestésico del binomio materno fetal en el proyecto EXIT para neonatos con hernia diafragmática. Rev Mex Anestesiol. 2021; 44 (2): 91-97. https://dx.doi.org/10.35366/99011
\end{abstract}

\begin{abstract}
RESUMEN. EXIT (tratamiento ex útero intraparto). El manejo anestésico para esta cirugía es significativamente diferente del manejo anestésico de una cesárea convencional, involucra anestesia general profunda con halogenados, administración de narcóticos, relajación uterina adecuada y preservación del flujo útero placentario hacia el feto durante la anestesia y al mismo tiempo tiene el beneficio de la anestesia general para que el producto obtenga anestesia y facilite el acceso a la vía aérea del neonato antes del pinzamiento del cordón umbilical. El fin de mantener la oxigenación a través de la placenta es efectuar la maniobra de intubación sin el riesgo de hipoxia. En el Hospital Infantil de México «Federico Gómez» se lleva a cabo este tipo de cirugías desde junio de 2007; sin embargo, aún no se cuenta con un manejo homogéneo. Material y métodos: A través de un reporte de casos se hizo una revisión perioperatoria de las pacientes embarazadas, a las cuales se les dio manejo anestésico para cirugía EXIT en el período comprendido entre junio de 2007 y mayo de 2018. Resultados: De los 43 casos manejados, la información anestésica perioperatoria obtenida permitió homologar el manejo anestésico del binomio materno fetal para poder realizar un protocolo intrahospitalario. Conclusión: El poder realizar un protocolo para el manejo anestésico del binomio materno fetal en el proyecto EXIT permite disminuir las complicaciones y la morbimortalidad ofreciendo mejor calidad en la atención.
\end{abstract}

ABSTRACT. The anesthetic management for this procedure is quite different from the anesthetic management of a conventional caesarean section. It includes deep general anesthesia with halogenated and narcotic administration, to get adecuate uterine relaxation and preservation of best uteroplacental flow to the fetus during anesthesia and the benefit of general anesthesia to aim the neonate show effects of of anesthetic medication by this way facilitate airway access before umbilical cord clamping. The aim to mantain placental oxigenation to neonate is avoid hypoxia risk during intubation technique. In the Hospital Infantil de Mexico «Federico Gómez» this procedures have been carried out since June 2007, however, there is still no homogeneous management. Material and methods: Through a case report, a perioperative files review was made of pregnant patients who were given anesthetic management for EXIT procedure in June 2007 to May 2018 period. Results: Of the 43 cases, the perioperative anesthetic information obtained allowed to standardize the anesthetic management of the maternal fetal binomial in order to perform an intrahospital protocol. Conclusion: The ability to perform a protocol for the anesthetic management of the maternal fetal binomial in the exit project allows to reduce complications and morbidity and mortality in the binomial, offering better quality of care.

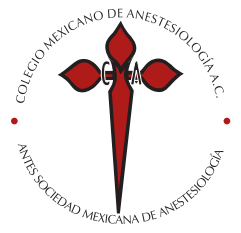

Palabras clave:

Procedimiento EXIT, intubación de neonato, anestesia para operación cesárea.

Keywords:

EXIT procedure, neonate intubation, cesarean section anesthesia, EXIT (ex utero intrapartum therapy).

\footnotetext{
* Jefe del Departamento de Anestesiología y Algología.

‡ Médico adscrito al Servicio de Anestesiología.

$\S$ Residente de Anestesiología Pediátrica del quinto grado.
}

Hospital Infantil de México «Federico Gómez».

Correspondencia:

Dr. Víctor Edmundo Fuentes-García Dr. Márquez 162, Colonia Doctores, México D.F. Tel. 55 5228-9917, ext. 2230 E-mail: vfuentesg@yahoo.com.mx

Recibido: 04-06-2019 Aceptado: 06-03-2020

\section{INTRODUCCIÓN}

$\mathrm{L}$ a mayoría de la literatura referente a procedimientos EXIT está limitada a reportes de caso y descripción de la técnica. Proporcionar anestesia para el binomio materno fetal con patología congénita del neonato es una práctica relativamente nueva; sin embargo, conforme van adquiriendo equipo y entrenamiento, más centros hospitalarios comienzan a cobrar auge para realizar dichos procedimientos.

EXIT (terapia ex útero intraparto) tiene el objetivo de realizar un procedimiento fetal al final de la gestación manteniendo un soporte útero placentario, donde la placenta sigue funcionando como órgano de respiración una vez que se obtiene el producto evitando la interrupción de la circula- 
ción útero placentaria inmediatamente, esto permite realizar intervenciones dentro de las cuales se encuentra el manejo avanzado de la vía aérea en el neonato.

EXIT es una intervención que permite llevar a cabo los procesos necesarios para la supervivencia fetal antes de pinzar la circulación útero placentaria y completar el parto. Era un procedimiento inicialmente empleado para casos con compromiso potencial de vía aérea y permitía la adecuada perfusión útero placentaria y la oxigenación del feto hasta establecer una vía aérea segura. Se utilizó principalmente para la reversión de la oclusión traqueal en el feto con diagnóstico prenatal de hernia diafragmática congénita severa que se había sometido a un tratamiento útero endoscópico oclusivo de la tráquea durante el embarazo ${ }^{(1,2)}$.

\section{Antecedentes}

EXIT, sigla anglosajona que traducida al español significa tratamiento ex útero intraparto, surge como modificación a la técnica Operating On Placental Support (OOPS). La técnica OOPS inicialmente describe la intervención para mantener el bypass útero placentario y poder realizar intervenciones en el feto $^{(3)}$. El primer informe publicado del procedimiento OOPS fue en 1989 por Norris, que destacaba el manejo de la vía aérea de un feto prematuro que tenía una masa cervical grande, diagnosticada prenatalmente como un teratoma cervical con mantenimiento de la circulación feto placentaria y varios intentos de intubación ${ }^{(4)}$. Langers describió el siguiente caso EXIT donde destacó la importancia de exteriorizar la mitad superior del cuerpo fetal para estabilizar la incisión y evitar la oclusión del cordón umbilical $^{(5)}$. Schwartz detalló dos procedimientos EXIT para un feto con hemangioma cervical y otro con micrognatia y teratoma orofaríngeo ${ }^{(6)}$. Tanaka describe una experiencia similar a la de Schwartz realizando el procedimiento EXIT en un feto con higroma quístico y monitoreo fetal adaptado utilizando Doppler en lugar de la oximetría de pulso ${ }^{(7)}$.

Mychaliska describió la primera serie de ocho casos exitosos con productos con vía aérea difícil. El autor fue el primero en acuñar el acrónimo EXIT para esta intervención; asimismo, desarrolló un abordaje sistematizado para el manejo de la vía aérea en los productos ${ }^{(8)}$.

La serie más grande publicada hasta la fecha es la de E. Lin y sus colegas, quienes describieron un total de 65 procedimientos EXIT a lo largo de 13 años. Esta descripción ha sido también la más completa del manejo anestésico hasta la fecha. Los casos se documentaron en el Hospital Infantil de Filadelfia de 1998 a 2011. Esta serie tuvo buenos resultados, reportó 14 complicaciones maternas correspondientes a atelectasia pulmonar, náusea y vómito postoperatorios, íleo, retención urinaria y dolor por analgesia posoperatoria deficiente, mismas que se resolvieron adecuadamente sin causar repercusiones a largo plazo ${ }^{(9)}$. El procedimiento EXIT también se ha realizado en embarazo gemelar ${ }^{(10,11)}$.

\section{Marco teórico}

Uno de los objetivos dentro del manejo anestésico para los procedimientos EXIT es optimizar la perfusión fetal manteniendo adecuada relajación uterina y al mismo tiempo asegurar la circulación y perfusión maternas ${ }^{(11)}$. Las anomalías que ocasionan compresión de la vía aérea tales como masa en cuello o torácicas son de especial interés para decidir la realización de procedimiento EXIT, ya que la morbimortalidad neonatal aumenta notablemente en los casos en los que se ve comprometida la vía aérea ${ }^{(12)}$.

Las indicaciones para realizar el procedimiento EXIT se han incrementado a través de los años y cobran vital importancia aquéllas en las cuales es importante asegurar la vía aérea. Entre los principales diagnósticos prenatales se encuentran los pacientes con masas gigantes en cuello, síndrome congénito de obstrucción de vía aérea alta, atresia laríngea, atresia traqueal, estenosis traqueal(13), o bien aquellos pacientes con diagnósticos en los cuales la dinámica pulmonar está comprometida tales como hipoplasia pulmonar, ejemplo de ello son las hernias diafragmáticas, secuestros pulmonares, malformaciones vasculares de la mitad superior del cuerpo, y algunas cardiopatías complejas ${ }^{(14)}$.

Generalmente los procedimientos EXIT se planean alrededor de las 37 semanas de gestación con la finalidad de mejorar el pronóstico del binomio.

La técnica anestésica marca que la madre se coloca en decúbito lateral izquierdo para disminuir los efectos de la compresión aortocava, sin comprometer el flujo hacia el útero y la placenta. La relajación uterina es un punto importante del manejo anestésico, ya que permite prevenir la separación prematura de la placenta y así mantener el flujo sanguíneo placento-fetal adecuado y su oxigenación, logrando dicha relajación con altas concentraciones de anestésicos halogenados. Recientemente ha tomado importancia la anestesia combinada, en la cual se utiliza la técnica de anestesia general balanceada y anestesia neuroaxial para lograr analgesia perioperatoria y postoperatoria.

Una vez que el neonato se obtiene, debe estar permeable la circulación útero placentaria, se realiza monitoreo con oximetría de pulso y se inicia instrumentación de la vía aérea; una vez asegurada, se interrumpe la circulación útero placentaria pinzando y seccionando el cordón umbilical, y se entrega al neonatólogo para la atención crítica al neonato. Se continúa el procedimiento con la madre y se previenen las complicaciones secundarias a la relajación uterina con el uso de ocitócicos y se evitan hemorragias ${ }^{(15-17)}$.

Todos estos eventos ocurren al mismo tiempo en un área quirúrgica, para lo cual se requiere del conocimiento tanto de la fisiología materna como de la fisiología del neonatal ${ }^{(18)}$. 
Tabla 1: Datos demográficos. $\mathrm{N}=43$.

\begin{tabular}{lc} 
Variable & Total \\
\hline Edad materna (años) & $29.5 \pm 6.51$ \\
Edad gestacional (semanas) & $36.74 \pm 0.44$ \\
Diagnóstico prenatal & \\
G1 + Hernia diafragmática & 9 \\
G1 + Hernia diafragmática congénita + RPM & 1 \\
G1 + MAV cerebral & 1 \\
G2 + Cardiopatía compleja & 1 \\
G2 + Hernia diafragmática + masa cervical & 1 \\
G2 + Hernia diafragmática congénita & 14 \\
G2 + Siameses isquiópagos & 1 \\
G2 + Siameses onfalópagos & 1 \\
G3 + Cardiopatía + masa intratorácica & 1 \\
G3 + Hernia diafragmática & 6 \\
G3 + Hernia diafragmática + MAV intratorácica & 1 \\
G3 + Hernia diafragmática + cardiopatía & 1 \\
G3 + Linfangioma cervical + cardiopatía & 1 \\
G4 + Hernia diafragmática + cardiopatía & 1 \\
G3 + Hernia diafragmática congénita izq. & 1 \\
G1 + Estenosis aórtica crítica & 1 \\
G1 + 40 SDG + atresia mitral + corazón izq. hipoplásico & 1 \\
\hline
\end{tabular}

Fuente: Hospital Infantil de México «Federico Gómez».

\section{MATERIAL Y MÉTODOS}

A través de un reporte de casos se realizó un estudio de tipo observacional, descriptivo y retrospectivo. Se hizo una revisión perioperatoria de las pacientes embarazadas a las cuales se les dio manejo anestésico para cirugía EXIT en el Hospital Infantil de México «Federico Gómez» en el período comprendido entre junio de 2007 y mayo de 2018. Se revisaron los expedientes de las pacientes programadas cuyos criterios de inclusión tomaron en cuenta el consentimiento informado para procedimiento anestésico, las condiciones del embarazo, patología del feto, técnica anestésica que se le otorgó a la madre, fármacos, dosis, farmacología, complicaciones así como sangrado, estado hemodinámico y dolor postoperatorio, además de aspectos relacionados con el producto tales como Apgar, semanas de gestación, patología, si requirió intubación, personal que realizó la intubación, número de intentos de la intubación y complicaciones.

Se analizaron los beneficios, ventajas y las complicaciones de los manejos establecidos para proponer, con base en la información obtenida, un protocolo de manejo anestésico homogéneo y así mejorar la calidad en la atención para ambos.

El objetivo de esta investigación es elaborar un protocolo de manejo anestésico perioperatorio homogéneo que permita establecer las pautas de tratamiento transanestésico de la madre y la atención especializada en la instrumentación de la vía aérea al neonato para pacientes que se someten a cirugía EXIT basándonos en la experiencia y práctica adquirida en el manejo de este tipo de pacientes en la institución con la hipótesis de que la elaboración de dicho protocolo incidirá en mejores resultados en la atención materno fetal. Para el análisis estadístico de las variables cuantitativas se utilizaron medidas de tendencia central y de dispersión, para variables nominales se utilizaron frecuencias y porcentajes.

\section{RESULTADOS}

En el período de junio de 2007 a mayo de 2018 en el Hospital Infantil de México «Federico Gómez» se atendieron en total 43 cesáreas con edad entre 18 y 42 años, media $29.5 \pm 6.51$, la edad gestacional fue desde 36.2 hasta 37.5 semanas de gestación con media de $36.74 \pm 0.44$, con diagnósticos de hernia diafragmática congénita, masas torácicas, cardiopatía compleja, masa cervical, linfangioma y gemelos siameses (Tabla 1).

Todas las pacientes fueron valoradas de manera preanestésica al momento de su llegada al hospital, cumpliendo de esta manera los criterios de inclusión. De la técnica anestésica $30(69.8 \%)$ fueron manejadas con anestesia general balanceada y anestesia neuroaxial y 13 (30.2\%) con anestesia neuroaxial única. Hay que hacer mención de que a todas las pacientes se les realizó el bloqueo neuroaxial aún despiertas (Tabla 2).

La decisión de la técnica anestésica, el empleo de narcóticos, inductores y relajantes neuromusculares fue por criterio de los anestesiólogos que atendieron la cirugía (Tabla 3).

Tabla 2: Técnica anestésica neuroaxial.

\begin{tabular}{|c|c|c|c|c|c|}
\hline Técnica anestésica & n (\%) & Técnica neuroaxial & n (\%) & $\begin{array}{c}\text { (Bupivacaína hiperbárica) } \\
\text { dosis }(\mu \mathrm{g} / \mathrm{kg})\end{array}$ & n (\%) \\
\hline AGB + neuroaxial & $30(69.8)$ & Espinal & $34(79.07)$ & 150 & $10(23.25)$ \\
\hline \multirow[t]{2}{*}{ Neuroxial } & $13(30.2)$ & Epidural + espinal & $9(20.93)$ & 200 & $29(67.44)$ \\
\hline & & & & 250 & 4 (9.31) \\
\hline Total n (\%) & $43(100)$ & & $43(100.00)$ & & $43(100.00)$ \\
\hline
\end{tabular}

Fuente: Hospital Infantil de México «Federico Gómez». 
De los 43 neonatos nacidos, a 39 se les realizó intubación endotraqueal. De estas intubaciones la mayoría fueron hechas por anestesiólogo pediatra (Tabla 4).

Para efectos de analgesia postoperatoria intravenosa se utilizaron dos esquemas establecidos con paracetamol y metamizol a dosis de $10 \mathrm{mg} / \mathrm{kg}$. Metamizol en 26 pacientes (60.47\%) y paracetamol en 17 pacientes (39.53). El esquema más utilizado mostró mayor satisfacción de analgesia postoperatoria en las pacientes.

Posterior al pinzamiento del cordón, se administraron ocitócicos, en su mayoría carbetocina en $100 \mu \mathrm{g}$ IV para 34 pacientes (79.07\%) y ocitocina con dosis de 10U IV en nueve pacientes (20.93\%), mostrando a la carbetocina con mayor efecto clínico para prevenir la hemorragia materna.

En relación al sangrado, se reporta que más de la mitad sangró $700 \mathrm{~mL} ; 22$ pacientes con sangrado de $700 \mathrm{~mL}$ (51.16\%); 14 pacientes con sangrado de $600 \mathrm{~mL}$ (32.56\%); cuatro pacientes con sangrado de $500 \mathrm{~mL}$ (9.3\%); y sólo tres pacientes con sangrado mayor de $700 \mathrm{~mL}$ con $6.98 \%$.

Con base en la información obtenida y la homogeneidad de los datos se deriva la siguiente conclusión:

\section{Protocolo de manejo anestésico materno y manejo anestésico del neonato}

La secuencia de intervención anestésica es:

1. La paciente se presenta al grupo de trabajo con el resumen de la evolución obstétrica y el manejo de intervención fetal en caso de que así haya sido.
2. El anestesiólogo evalúa a la paciente embarazada físicamente el día anterior a su cesárea, apegándose a los criterios médicos y legales instituidos para la evaluación preanestésica, tomando en cuenta el estado físico, los antecedentes, los laboratorios, los estudios radiológicos, y en especial la atención prenatal a la madre y al producto. Se dan indicaciones que incluyen ayuno preoperatorio mayor de ocho horas y canalizar vena con un catéter $18 \mathrm{~g}$.

\section{Día de la cirugía}

En el área de preanestesia se efectúa una nueva evaluación corroborando los datos obtenidos en la valoración preanestésica. En quirófano se realiza monitoreo no invasivo con electrocardiograma, presión arterial no invasiva, oximetría, temperatura y capnografía.

\section{Manejo de la madre}

La técnica anestésica es anestesia combinada como se detalla a continuación:

- Accesar en total dos vías venosas de calibre no menor de $18 \mathrm{~g}$.

- Colocación de bloqueo subaracnoideo para analgesia postoperatoria empleando:

bupivacaína hiperbárica al 5\% a dosis de $200 \mu \mathrm{g} / \mathrm{kg}$; alcanzando con estas dosis niveles de T11, T12. Ésta técnica

\begin{tabular}{|c|c|c|c|c|c|c|c|c|c|}
\hline $\begin{array}{l}\text { Fentanyl. dosis } \\
\bar{x}(\mu \mathrm{g} / \mathrm{kg})\end{array}$ & n (\%) & $\begin{array}{l}\text { Propofol dosis } \\
\bar{x}(\mu \mathrm{g} / \mathrm{kg})\end{array}$ & n (\%) & $\begin{array}{l}\text { Midazolam dosis } \\
\bar{x}(\mu \mathrm{g} / \mathrm{kg})\end{array}$ & n (\%) & $\begin{array}{l}\text { Relajante dosis } \\
\overline{\bar{x}}(\mu \mathrm{g} / \mathrm{kg})\end{array}$ & n (\%) & Sevofluorane & n (\%) \\
\hline 3 & $24(80)$ & 3 & $23(77)$ & 100 & $9(30)$ & Cis. Atrac. (200) & $22(51.16)$ & CAM 0.7 & $8(18.60)$ \\
\hline \multirow[t]{3}{*}{4} & $6(20)$ & 4 & $7(23)$ & & & Vecuronio (100) & 5 (11.63) & CAM 0.8 & $20(46.51)$ \\
\hline & & & & & & Rocuronio (600) & $3(6.98)$ & CAM 1.0 & $2(4.66)$ \\
\hline & 30 (100) & & $30(100)$ & & & & $30(100)$ & & $30(100)$ \\
\hline
\end{tabular}

Fuente: Hospital Infantil de México «Federico Gómez».

\begin{tabular}{cccc} 
& & Tabla 4: Intubación al neonato. \\
Neonato & $\mathrm{n}(\%)$ & Intubación & $\mathrm{n}(\%)$ \\
\hline Con intubación & $39(90.7)$ & Por neonatólogo intentos & $8(20.51)$ \\
Sin intubación & Por anestesiólogo & $31(79.49)$ & 1 \\
Total & $4(9.3)$ & & \\
\hline
\end{tabular}

Fuente: Hospital Infantil de México «Federico Gómez». 


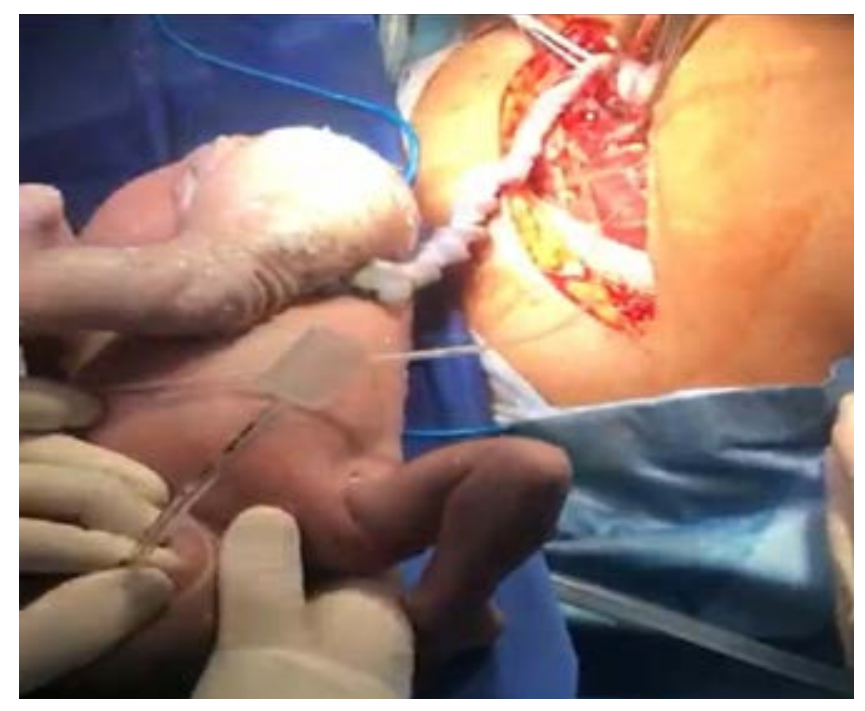

Figura 1: Intubación de neonato por anestesiólogo.

debe hacerse con la paciente despierta para facilitar el posicionamiento y hemodinamia de la madre y el producto. Una vez bloqueada se procede a instalación de la anestesia general.

- Se debe manejar con técnica para estómago lleno. Inducción e intubación de secuencia rápida.

- Inducción propofol (3 mg/kg), fentanyl ( $3 \mu \mathrm{g} / \mathrm{kg})$, relajante cisatracurio $(200 \mu \mathrm{g} / \mathrm{kg})$, intubación orotraqueal y el mantenimiento con sevofluorano 0.8 CAM.

En caso de presentarse hipotensión materna, debe manejarse con reanimación hídrica con Sol Ringer de Lactato iniciando con $10 \%$ de volumen sanguíneo circulante y uso de vasopresores (efedrina en bolos, utilizando bolos de $5 \mu \mathrm{g}$ ) con monitoreo estricto de la presión arterial no invasiva.

El abordaje de la vía aérea de la madre se realiza a través de la intubación orotraqueal, con el objeto de mantener oxigenación adecuada principalmente durante el tiempo que transcurre la histerotomía y el pinzamiento del cordón umbilical; oxigenación que debe ubicarse entre 21 y $80 \%$ de acuerdo a la respuesta en la $\mathrm{spO}_{2}$ periférica de la paciente, lo cual permitirá oxigenación adecuada al producto ${ }^{(19)}$.

- El halogenado recomendado por sus características farmacológicas y farmacocinéticas en este tipo de procedimientos es el sevofluorano en concentraciones de 0.8 CAM.

Posterior al nacimiento se administran medicamentos ocitócicos para la recuperación del tono uterino y se continúa con el manejo anestésico a la manera habitual.

- Analgesia bolo metamizol $10 \mathrm{mg} / \mathrm{kg}$ vía intravenosa.

\section{Manejo de la vía aérea del neonato}

Material estéril: a) Mango de laringoscopio mini, hojas rectas Miller número 0 y número 1 , guía de intubación pediátrica desechable nueva, sondas endotraqueales sin globo y con globo número 2.5, 3.0, 3.5 y 4.0 mm de diámetro interno. b) Dispositivo para manejo de la vía aérea difícil: dispositivos supraglóticos (mascarilla laríngea, cánula de guedell, mascarilla facial), fibroscopio pediátrico, videolaringoscopio de acuerdo a la edad y c) Circuito para administrar presión positiva tipo bolsa mascarilla reservorio y oxígeno suplementario.

Técnica: la intubación del recién nacido se hace con la técnica habitual para manejo de vía aérea difícil en neonato con el producto en las extremidades inferiores de la madre con el cordón umbilical permeable. Debe hacerse por el anestesiólogo experto, ya que es la persona con más práctica en el manejo de la vía aérea para evitar retraso en conseguir la intubación (Figura 1).

Una vez asegurada la vía aérea se corrobora la entrada y salida de aire bilateral por auscultación de ambos hemitórax, y se procede a ligar y seccionar el cordón umbilical.

Ya asegurada la vía aérea se traslada a la cuna térmica dentro del mismo quirófano para su atención por los especialistas en medicina crítica del neonato.

\section{DISCUSIÓN}

La cesárea con técnica EXIT ha sido poco estudiada y los reportes que existen son escasos; sin embargo, la oportunidad de recabar la información de manera retrospectiva nos permitió realizar un análisis a través de los años con la experiencia clínica que se tiene en el Hospital Infantil de México «Federico Gómez» para elaborar un protocolo de manejo anestésico homogéneo. Siempre beneficiando al binomio madre e hijo en diferentes aspectos como mantener adecuados parámetros hemodinámicos, analgesia posoperatoria adecuada para la madre y la intubación oportuna del producto para disminuir las complicaciones en el manejo de la vía aérea.

El objetivo inicial del estudio era la atención prenatal a hernia diafragmática congénita con y sin intervención fetal, posteriormente se amplió a diversas anomalías congénitas como tumoraciones, cardiopatías, embarazos gemelares, malformaciones vasculares, atresias y agenesias pulmonares.

Durante los últimos años, los avances al diagnosticar y tratar los defectos congénitos y prevenir la mortalidad neonatal han sido impresionantes ${ }^{(9)}$. La importancia de la atención al binomio en el mismo centro hospitalario disminuye el período de enorme riesgo que representa el traslado evitando estados de hipotermia, intubación precipitada, aumento de posibilidad de infección y además se brinda pronta atención al neonato. Motivo por el cual se desarrolla la cirugía fetal así como la cirugía EXIT con el propósito de asegurar la vía aérea al mo- 
mento del nacimiento, mejorar las condiciones del neonato e incrementar sus probabilidades de supervivencia, siendo importante la unificación de los criterios de manejo anestésico para continuar tratando a los pacientes bajo la modalidad de binomio materno fetal con una mayor tasa de éxito ${ }^{(12,13)}$.

Resulta importante que el diagnóstico prenatal sea oportuno, y que se realice con la mayor certeza posible ${ }^{(14)}$. Se ha demostrado que la intervención fetal cambia el pronóstico de manera positiva, comparado con conducta expectante mejorando la sobrevida en más del doble en algunos casos, el mejor ejemplo de ello es la oclusión traqueal por fetoscopía en los pacientes con hernia de Bochdalek antes de la semana 26.

El seguimiento del binomio a través de las semanas y la comunicación constante del equipo médico multidisciplinario permite una planeación adecuada para el momento de la intervención EXIT.

El hecho de establecer una adecuada relación médicopaciente durante la entrevista para la obtención de datos patológicos y no patológicos es fundamental, igualmente las condiciones que rodearon a la gestación es un punto muy importante para establecer la confianza de la paciente con el anestesiólogo así como explicar los pasos que se seguirán desde el momento de su llegada al hospital hasta el momento en que termine la intervención. Diversos autores en sus investigaciones relacionadas a este tema reconocen la importancia de mantener un trabajo de equipo, ya que se trata de una tarea multidisciplinaria entre obstetras, cirujanos pediatras, anestesiólogos pediatras y neonatólogos determinando que con una estrategia cuidadosa y planeada, además de un adecuado monitoreo, la cirugía EXIT permite que el equipo cambie las condiciones de una paciente que es potencialmente inestable a estable ${ }^{(6,8)}$.

El manejo anestésico del EXIT se basa en el uso de concentraciones de halogenado suficientes para facilitar la relajación uterina durante la cesárea, además de asegurar la vía aérea del producto durante el mantenimiento de un bypass útero placentario hasta que se realice el corte del cordón umbilical. Lo anterior con mínimas complicaciones materno fetales. En nuestro estudio la técnica anestésica que mejores resultados arrojó fue la combinada, basándose en bloqueo neuroaxial espinal, permitiendo adecuada sinergia con el halogenado. Schwartz y Mychaliska hicieron hincapié en que el principio central del procedimiento EXIT era controlar la hipotonía uterina para preservar la circulación útero placentaria, misma que documentaron incluso hasta por 60 minutos sin provocar atonía uterina o hemorragia obstétrica. El manejo de la vía aérea en el producto se ha asegurado en varios estudios a través del uso de broncoscopía, intubación orotraqueal, traqueostomía y traqueostomía con intubación retrógrada, siendo la más frecuente la intubación orotraqueal como en nuestro estudio ${ }^{(6,8)}$.

Para la inducción anestésica, en su mayoría no fue necesario utilizar benzodiacepinas y las dosis de fentanilo a 3 $\mu \mathrm{g} / \mathrm{kg}$ fueron adecuadas para conservar la relajación uterina óptima manteniendo un adecuado flujo placenta-producto. El halogenado sevofluorane se recomienda por su fácil y predecible resultado para alcanzar relajación uterina, además de proporcionar mínima depresión fetal. Punto importante es vigilar la dosificación de agentes volátiles, ya que CAM altos se asocian a depresión miocárdica y bradicardia fetal, asimismo puede condicionar relajación uterina después de extraer al producto ${ }^{(10)}$. En nuestra revisión observamos que con valores de un CAM 0.8 obtuvimos resultados favorables en cuanto a la estabilidad cardíaca del neonato.

Dentro de los ocitócicos utilizados, la carbetocina demostró menor sangrado y adecuada tonicidad uterina durante el lapso de cordón permeable comparado con la ocitocina. No se presentaron complicaciones por hemorragias obstétricas ni intervenciones adicionales por dichas situaciones y tampoco fue necesario el uso de hemoderivados, resultados muy similares a los que se describen en otros reportes de caso y revisiones de la literatura ${ }^{(20)}$.

Para la analgesia postoperatoria, el uso de analgésicos a nivel central permite adecuada analgesia postoperatoria, y adiciona los efectos del bloqueo neuroaxial a dosis recomendadas y se observa que la tasa de hipotensión materna, náusea y vómitos postoperatorios no es alta con dichas dosis y con el empleo de los analgésicos mencionados se logra un control analgésico adecuado para el postoperatorio inmediato. Algunos autores mencionan el uso de analgesia multimodal con uso de anestésicos locales, administración de opioides y analgésicos no esteroideos ${ }^{(21,22)}$. En nuestro estudio fue suficiente la administración de paracetamol y metamizol a dosis de $10 \mathrm{mg} / \mathrm{kg}$. En relación con las complicaciones postoperatorias reportadas en nuestro estudio sólo en dos pacientes se presentó náusea y vómito asociados a hipotensión, las cuales remitieron con la administración de carga hídrica de solución Hartmann a 20 mL/kg y administración de ondansetrón a 100 $\mu \mathrm{g} / \mathrm{kg}$, complicaciones inferiores a las descritas en la literatura médica. E. Lin en su estudio reportó 14 complicaciones maternas correspondientes a atelectasia pulmonar, náusea y vómito postoperatorios; íleo, retención urinaria y dolor por analgesia postoperatoria deficiente resueltas adecuadamente sin causar repercusión a largo plazo. Punto importante es mantener una adecuada hemodinamia en el manejo anestésico materno, considerando la premisa de que son dos pacientes que se someten a procedimiento quirúrgico y anestésico simultáneamente, de esta manera las directrices del manejo están dirigidas a mantener condiciones óptimas materno-fetales.

Dentro de los puntos más importantes destaca el aseguramiento de la vía aérea del neonato, misma que se ha demostrado debe realizarse por el anestesiólogo pediatra, especialista con mayor experiencia en la instrumentación de la vía aérea en estos pacientes. Hubo necesidad de hacer cambio de sonda endotraqueal en cinco pacientes, ya 
que se presentaba fuga y el anestesiólogo pediatra hizo el cambió al tamaño adecuado sin ninguna complicación y al primer intento.

El presente estudio con un diseño de reporte de casos tiene debilidades metodológicas, pero nos da la pauta para poder llevar a cabo estudios a futuro con diferente diseño metodológico, incluso llegar a realizar ensayos clínicos con intervenciones más precisas o específicas. Es necesario para poder tener mayor impacto ampliar el tamaño de la «n», lo cual nos obliga a continuar este estudio.

\section{CONCLUSIÓN}

Unificar los criterios del manejo anestésico del binomio para procedimiento EXIT en neonatos con hernia diafragmática o alguna otra malformación permitió la elaboración de una guía intrahospitalaria, con el objetivo de tener mayor conocimiento de la patología, técnicas anestésicas alternativas, disminuir las complicaciones y la morbimortalidad en el binomio, y tener la posibilidad de poder reproducirse en otros centros hospitalarios.

\section{REFERENCIAS}

1. Restrepo CE, Gomez ME, Puerta JJ, Upegui A. 2006. Anaesthesia for the EXIT procedure: the value of fast track anaesthesia. International Journal of Obstetric Anesthesia. 2006;15:133-138.

2. Kodali BS, Bharadwaj S. Foetal surgery: Anaesthetic implications and stratetegic management. Indian J Anaesth. 2018; 62: 717-723.

3. Kumar K, Miron C, Indu SS. Maternal anesthesia for EXIT procedure: A systematic review of literature. J Anesthesiol Clin Pharmacol. 2019;35:19-24.

4. Norris MC, Joseph J, Leighton BL. Anesthesia for perinatal surgery. Am J Perinatol. 1989;6:39-40.

5. Langer JC, Fitzgerald PG, Desa D, Filly RA, Golbus MS, et al. Cervical cystic hygroma in the fetus: clinical spectrum and outcome. J Pediatr Surg. 1999;25:58-62.

6. Schwartz MZ, Silver H, Schman S. Maintenance of the placental circulation to evaluate and treat an infant with massive head and neck hemangioma. J Pediatr Surg. 1993;28:520-522.

7. Tanaka M, Sato S, Naito H, Nakayama H. Anesthetic management of a neonate with prenatally diagnosed cervical tumour and upper airway obstruction. Can J Anaesth. 1994;41:236-240.

8. Mychaliska GB, Bealer JF, Graf JL, Rosen MA, Scott Adzick N, et al. Operating on placental support; the ex utero intrapartum treatment (EXIT) procedure. J Pediatr Surg. 1997;32:227-231.

9. Lin EE, Moldenhauer JS, Tran KM, Cohen DE, Scott AN. Anesthetic management of 65 cases of ex utero intrapartum therapy: A 13 Year Single Center Experience. Anesth Analg. 2016;30:1-9.

10. Liechty KW, Crombleholme TM, Weiner S, Bernick B, Flake AW, et al. The ex utero intrapartum treatment procedure for a large fetal neck mass in a twin gestation. Obstet Gynecol. 1999;93:824-825.

11. Midrio P, Zadra N, Grismondi G, Suma V, Pitton MA, Salvadori S, et al. EXIT procedure in a twin gestation and review of the literature. Am J Perinatol. 2001;18:357-362.
12. Pucher B. Szydlowski J, Jonczyk-Potoczna K, Srocznski J. The EXIT (ex-utero intrapartum treatment)- procedure- from the paediatric ENT perspective. Acta Otorhinolaryngol Ital. 2018;38:480-484.

13. Yuan Shi-Min The application of ex utero intrapartum treatment (EXIT) procedure for cardiothoracic disorders. Signa Vitae. 2018;14:14-16.

14. Laje P, et al., Inmediate operative management of the fetus with airway anormalies resulting of malformations. Semin Fetal Neonatal Med. 2016;21:240-5.

15. Myers LB, Bulich LA. Anesthesia for ex utero intrapartum treatment exit procedure. In: Myers LB, Bulich LA. Anesthesia for fetal intervention and surgery. Hamilton, Ontario: Editorial BC Decker Inc.; 2005. pp. 145-156.

16. Tran KM, Maxwell LG, Cohen DE, Adamson PC, Moll V, Kurth CD, Galinkin JL. Quantification of serum fentanyl concentrations from umbilical cord blood during ex utero intrapartum therapy. Anesth Analg 2012;114:1265-1267.

17. Van de Velde M, De Buck F. Fetal and maternal analgesia/anesthesia for fetal procedures. Fetal Diagn Ther. 2012;31:201-209.

18. Lin EE, Tran KM. Anesthesia for fetal surgery. Semin Pediatr Surg. 2013;22:50-55.

19. Abraham RJ, Sau A, Maxwell D. A review of the EXIT (Ex utero Intrapartum Treatment) procedure. J Obstet Gynaecol. 2010;30:1-5.

20. Nnamani Nwamaka. From OOPS to EXIT: a review of the origins and progression of ex utero intrapartum treatment. Journal of Anesthesia and Clinical Research. 2015;6:540-545.

21. Ospina García Juan Camilo, Wuesthoff Prieto Carolina, Eslava Cerón Santiago. EXIT: tratamiento exútero intraparto. Reporte de un caso y revisión de la literatura. Revista Colombiana de Obstetricia y Ginecología. 2012;63:155-162.

22. Sviggum HP, Kodali BSr. Maternal anesthesia for fetal surgery. Clin Perinatol. 2013;40:413-427. 